\title{
PREVISÃO DE DEMANDA NA INDÚSTRIA DE PETRÓLEO: UM ESTUDO DE CASO NO ESTADO DO ESPÍRITO SANTO
}

\section{DEMAND FORESCASTING IN THE OIL INDUSTRY: A CASE STUDY IN THE STATE OF ESPÍRITO SANTO}

\author{
Noéle Bissoli Perini ${ }^{1}$; Adelmo Inácio Bertolde ${ }^{2}$ \\ ${ }^{1}$ Universidade Federal do Espírito Santo - UFES - Espírito Santo - ES - Brasil \\ noeleperini@hotmail.com \\ ${ }^{2}$ Universidade Federal do Espírito Santo - UFES - Espírito Santo - ES - Brasil \\ adelmoib@gmail.com
}

\begin{abstract}
Resumo
A previsão de demanda se tornou um dos principais fatores na gestão das empresas, revelando-se como uma alternativa estratégica para enfrentar as oscilações da demanda e, assim, evitar prejuízos. A indústria de petróleo possui grande importância para a economia nacional, contribuindo com a geração de empregos diretos e indiretos. Por isso, estudos sobre o consumo dos derivados combustíveis de petróleo auxiliam nas projeções e planejamentos desse setor. Além de que a previsão de vendas é uma importante ferramenta gerencial. Desta forma, este artigo tem como objetivo ajustar um modelo de previsão da demanda, mais adequado à demanda de gasolina no estado do Espírito Santo, com base nos dados de consumo mensais do período de janeiro de 2000 à março de 2015. Para a realização deste estudo primeiramente foi apresentada a revisão bibliográfica dos principais tipos e técnicas de previsão de demanda. Posteriormente, empregaram-se técnicas quantitativas de previsão da demanda (média móvel, média exponencial móvel, equação linear para a tendência, ajustamento exponencial para a tendência, sazonalidade simples e sazonalidade com tendência), e por meio dos parâmetros referentes ao erro acumulado e Desvio Médio Absoluto (MAD), compararam-se os métodos, e foi possível identificar que as principais técnicas de previsão foram a Média Exponencial Móvel e o Ajustamento Exponencial para a Tendência.
\end{abstract}

Palavras-chave: previsão de demanda; gasolina; tendência.

\section{Introdução}

As mudanças econômicas ocorridas nos últimos tempos como o processo de globalização, têm forçado as organizações empresarias a adaptarem-se continuamente para enfrentar os desafios de manterem-se no mercado de forma competitiva. Esta situação requer especial atenção das empresas ao planejamento adequado das atividades envolvidas no processo de produção, a fim de melhorar o fluxo e alocação de informações, materiais e pessoas, e atender satisfatoriamente à demanda pelos seus produtos (CAVALHEIRO, 2003). 
Neste contexto, a previsão de demanda se tornou um dos principais fatores na gestão das empresas, revelando-se como uma alternativa estratégica para enfrentar as oscilações da demanda e, assim, evitar prejuízos (MANCUSO; WERNER, 2014).

A previsão da demanda é uma ferramenta que possibilita o planejamento estratégico da produção, da comercialização, das finanças e a formulação de políticas dentro do setor. Partindo deste ponto, os gestores recebem informações privilegiadas sobre possíveis variações no mercado, com isso, adequando suas ações para melhor atender as necessidades do mercado, como qualidade ou quantidade (TUBINO, 2009).

A indústria de petróleo possui grande importância para a economia nacional, contribuindo com a geração de empregos diretos e indiretos. Por isso, estudos sobre o consumo dos derivados combustíveis de petróleo auxiliam nas projeções e planejamentos desse setor. Além de que a previsão de vendas é uma importante ferramenta gerencial.

Para tanto, o Portal Brasileiros de Dados Abertos, do Governo Federal, disponibiliza informações acerca destes derivados combustíveis de petróleo, tais como, etanol hidratado, gasolina C, gasolina de aviação, GLP, óleo combustível, óleo diesel, querosene de aviação e querosene iluminante de todo o território nacional.

Portanto, esta pesquisa busca responder a seguinte questão: Qual modelo de previsão de demanda melhor se adequa a demanda de gasolina, no estado do Espírito Santo?

O objetivo geral desta monografia é ajustar um modelo de previsão da demanda de gasolina no estado do Espírito Santo.

Este artigo está organizado em seis seções, iniciando-se com a presente introdução. Na seção 2 é apresentada a revisão de literatura sobre previsões e tipos de previsões. A seção 3 aborda os conceitos relativos à Previsão de Demanda, tais como, Etapas de um modelo de previsão e Técnicas de previsão. Na seção 4, a metodologia é descrita. Já, a seção 5 apresenta os resultados do estudo de caso. Por fim, na seção 6 as conclusões e recomendações são apresentadas.

\section{Previsões}

Prever é a arte e a ciência de predizer eventos futuros. Isso pode envolver a utilização de dados históricos e a sua projeção no futuro com algum tipo de modelo matemático. Boas previsões são uma parte essencial de operação eficiente de serviços e manufatura. Elas também são um importante recurso de modelagem na tomada de decisões estratégicas e táticas. Uma previsão pode ser classificada pelo horizonte de tempo futuro que ela cobre, sendo eles (HAIZER e RENDER, 2001): 
- Previsão de curto prazo: esta previsão tem uma abrangência de tempo de até um ano, mas geralmente menor que três meses. É utilizada para planejamento de compras, programação de tarefas, níveis de força de trabalho, alocação de tarefas e níveis de produção.

- Previsão de médio prazo: uma previsão de médio prazo, ou intermediária, geralmente se estende por 3 meses a 3 anos. É útil para planejamento de vendas, planejamento e orçamentos de produção, orçamento financeiro e análise de diversos planos de operação.

- Previsão de longo prazo: estendendo-se geralmente por três anos ou mais, as previsões de longo prazo são utilizadas no planejamento de novos produtos, gastos de capital, localização ou expansão de instalações e pesquisa e desenvolvimento.

As previsões de médio e longo prazos distinguem-se das de curto prazo por três aspectos:

a) As previsões de prazos intermediário e longo tratam de questões mais abrangentes e suportam decisões gerenciais referentes a planejamento e produtos, fábricas e processos;

b) A previsão de curto prazo normalmente emprega tecnologias diferentes das previsões de maior alcance. Técnicas matemáticas, como médias móveis, o suavizamento exponencial e a extrapolação de tendências, são comuns nas projeções de curto prazo. Métodos mais abrangentes, menos quantitativos, são úteis na previsão de questões tais como se um novo produto deve ser introduzido na linha de produtos de uma empresa;

c) As previsões de curto prazo tendem a ser mais exatas do que as de longo prazo. Os fatores que influenciam a demanda se modificam a cada dia. Desse modo, à proporção que o horizonte de tempo se estende, é provável que a exatidão de uma previsão diminua. Portanto, as previsões de vendas devem ser atualizadas regularmente para que seu valor e integridade sejam mantidos. Após cada período de vendas, as previsões devem ser revistas e verificadas.

\subsection{Tipos de Previsões}

As organizações utilizam três tipos principais de previsões no planejamento de futuras operações (HAIZER e RENDER, 2001):

- Previsões econômicas: tratam do ciclo do negócio, prevendo taxas de inflação, fontes de capital, início de construção de projetos habitacionais e outros indicadores de planejamento.

- Previsões tecnológicas: relacionam-se a índices de progresso tecnológico, que podem resultar na criação de excelentes produtos novos, exigindo novas fábricas e novos equipamentos.

- Previsões de demanda: são projeções da demanda pelos produtos e serviços de uma empresa. Essas previsões, também chamadas de previsões de vendas, dirigem a produção, a capacidade e os sistemas de programação da empresa e servem como inputs para o planejamento financeiro, de marketing e de pessoal. 
A ênfase desta pesquisa será a previsão de demanda.

\section{Previsão de Demanda}

\subsection{Etapas de um modelo de previsão}

Um modelo de previsão de demanda pode ser dividido em cinco etapas básicas, conforme apresentado na Figura 1.

Figura 1 - Etapas do modelo de previsão da demanda

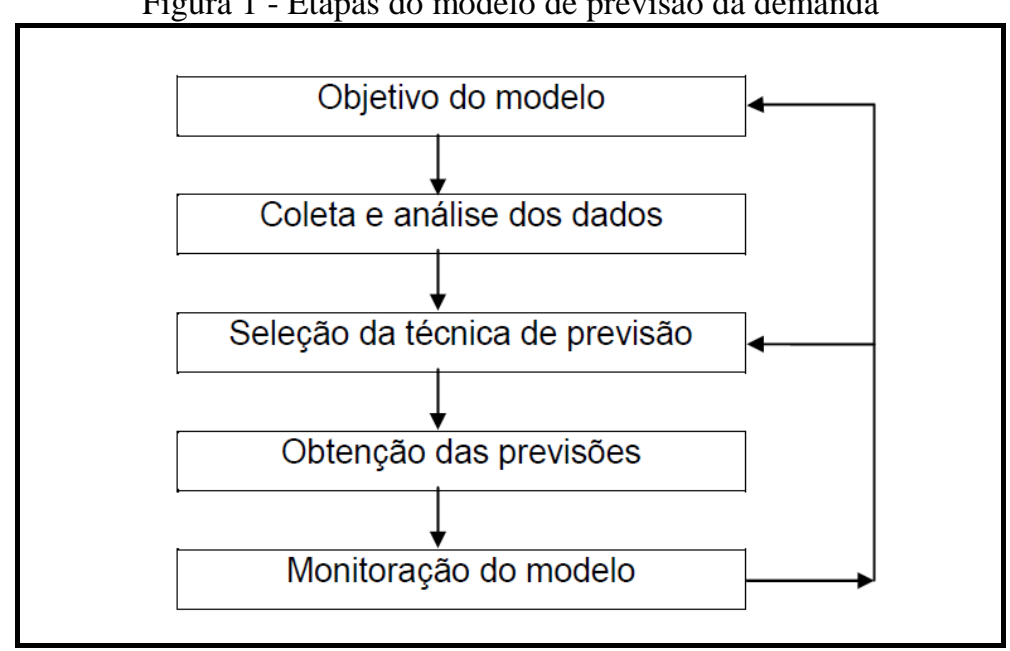

Fonte: Tubino (2009)

Primeiramente é definido o objetivo do modelo, e com base nessa decisão são coletados e analisados os dados, para selecionar a técnica de previsão mais apropriada. Dessa forma, calcula-se e obtém-se a previsão da demanda e, como forma de feedback, monitoram-se e atualizam-se os parâmetros empregados no modelo por meio da análise do erro de previsão.

\subsection{Técnicas de previsão}

Corrêa e Corrêa (2007) dividem as técnicas que tratam as informações disponíveis em duas abordagens (Figura 2): as abordagens quantitativas (baseadas em séries históricas projetadas para o futuro segundo algum método) e as abordagens qualitativas (baseadas em fatores subjetivos ou de julgamento). Em geral, os modelos quantitativos necessitam de uma extensa base de dados histórica, para que por meio dela se possam identificar os padrões de comportamento que serão projetados para o futuro. Dessa forma, percebe-se que são mais úteis para, por exemplo, fazer previsões de demanda de produtos mais maduros, que estejam há mais tempo no mercado. Já os modelos qualitativos encaixam-se melhor em previsões de produtos novos ou lançamentos, para os quais não há históricos longos. Outro fator determinante para a "ponderação" do uso de modelos quantitativos e qualitativos na geração de uma previsão é o horizonte da previsão. Em geral, quanto maior o horizonte de previsão, menos válida é a hipótese de que os padrões do passado se repetirão 
no futuro (uma hipótese que se assume quando se usam modelos mais quantitativos). Isso significa que modelos quantitativos tendem a ser mais adequados a previsões de curto prazo.

Figura 2 - Abordagens para métodos de tratamento de informações em previsões

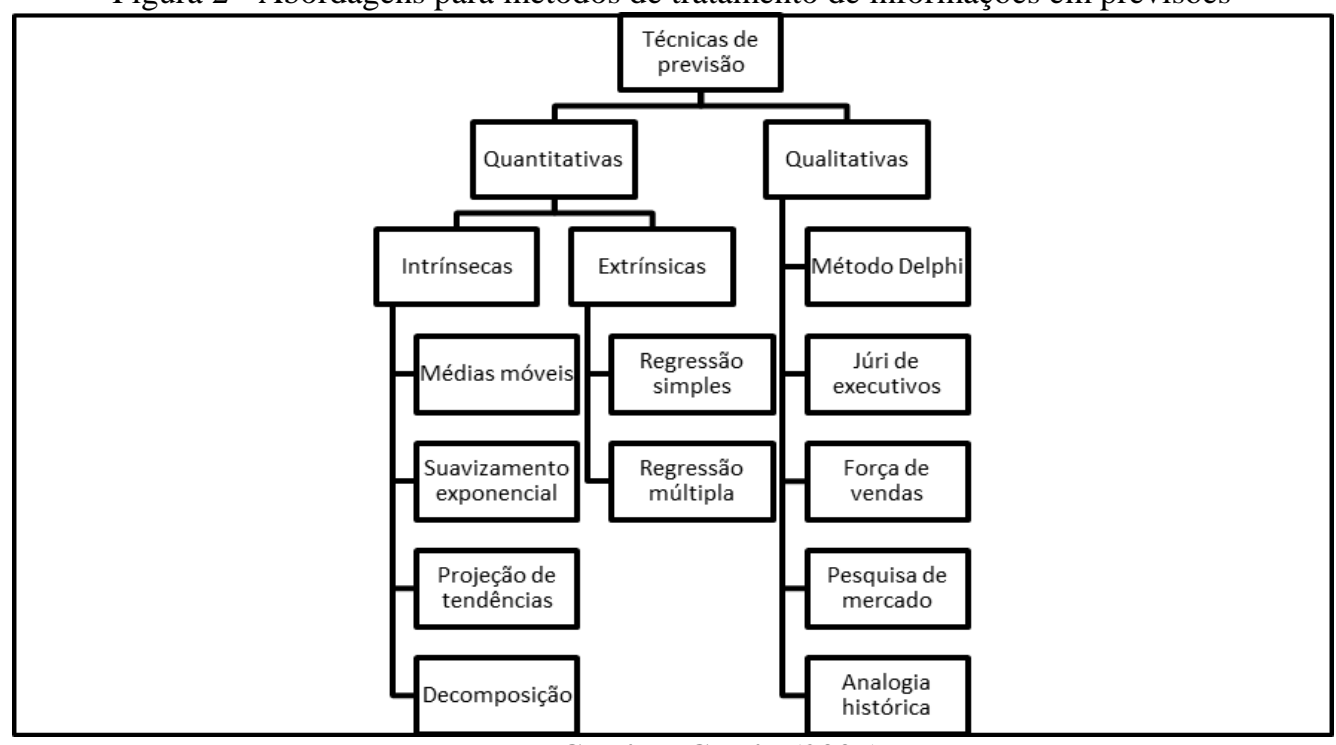

Fonte: Corrêa e Corrêa (2007)

\subsubsection{Métodos Qualitativos}

Os métodos qualitativos são geralmente utilizados e recomendados para quando não existem dados históricos como base para as previsões (PEINADO; GRAEML, 2007). A seguir são explicitados cada um dos métodos qualitativos apresentados na Figura 2.

a) Método Delphi: a técnicas procura obter uma opinião de grupo por meio de um processo anônimo de feedback interativo controlado. O método usual submete questionários a um grupo de especialistas. A informação do grupo é reunida, resumida, agregada e apresentada anonimamente como feedback ao grupo. Cada membro do grupo pode então comparar sua própria previsão com a "média" do grupo. O indivíduo pode então modificar, ou não, sua primeira previsão e o processo é repetido. Por fim, um consenso do grupo emerge (SLACK et al., 2002);

b) Júri de executivos: esse método procura captar a opinião de pequenos grupos, em geral, de executivos de nível alto sobre alguma variável que se pretende prever. É frágil no sentido de permitir viés e não se pode assumir a priori que a estimativa obtida representa consenso do grupo (CORRÊA; CORRÊA, 2007);

c) Força de vendas: cada vendedor estima como serão as vendas em sua região. Essas previsões depois são revistas para assegurar que sejam realistas. Em seguida, são combinadas nos níveis distrital e nacional para se chegar a uma previsão geral (HEIZER; RENDER, 2001);

d) Pesquisa de mercado: este método solicita informações de clientes ou de clientes potenciais quanto aos futuros planos de compra (HEIZER; RENDER, 2001); 
e) Analogia histórica: este método procura identificar produtos similares dos quais se possuem dados para, por analogia, melhor estimar, por exemplo, um produto novo (CORREA; CORRÊA, 2007).

\subsubsection{Métodos Quantitativos}

As técnicas quantitativas consistem em analisar os dados passados objetivamente, empregando-se modelos matemáticos para projetar a demanda futura. Elas podem ser subdivididas em dois grupos: as técnicas baseadas em séries temporais, e as técnicas baseadas em correlações (TUBINO, 2009).

\subsubsection{Previsões baseadas em séries temporais}

Para Bruni (2007) dados estatísticos que apresentam a evolução de valores ao longo do tempo são chamados de séries temporais. Geralmente, qualquer atividade de negócios costuma registrar e armazenar grupos distintos de séries temporais, associadas às diferentes variáveis, por exemplo, dados diários, semanais, mensais de números relativos a vendas, custos, lucros, estoques e outros. As séries temporais podem ser decompostas em quatro grandes componentes: tendência, variações cíclicas, variações sazonais e variações irregulares ou aleatórias.

a) Tendência: descreve um movimento suave, a longo prazo, dos dados, para cima ou para baixo. $\mathrm{Na}$ análise de séries temporais, existem dois objetivos principais associados ao isolamento das tendências. O primeiro objetivo envolve a identificação da tendência com o objetivo de uso em previsões. O segundo objetivo envolve a sua remoção de forma a permitir o estudo dos outros componentes da série temporal;

b) Variações cíclicas: correspondem a um certo grau de regularidade a longo prazo no comportamento das séries temporais. Por exemplo, o aumento das vendas de bandeiras do Brasil em função da copa do mundo de futebol é uma variação cíclica que ocorre a cada quatro anos;

c) Variações sazonais: representam regularidades de variações na série em períodos curtos de tempo, geralmente dentro de ano. Por exemplo, vendas de ovos de páscoa são aquecidas nos meses entre março e abril;

d) Variações irregulares ou aleatórias: correspondem a ruídos na série temporal em decorrência de fatores variados. Como são aleatórios, não são previstos nos modelos.

O processo tradicional de análise de séries temporais envolve a decomposição dos componentes e a análise individual de cada um deles. Em seguida, os componentes são combinados a fim de se observar o efeito conjunto. Duas formas costumam ser empregadas na decomposição das séries nos seus componentes (BRUNI, 2007): 
a) Forma aditiva: considera que a série temporal é uma soma dos quatro componentes;

b) Forma multiplicativa: considera que a série temporal é uma soma dos quatro componentes.

Para Tubino (2009) as previsões que utilizam séries temporais partem do pressuposto de que a demanda futura não é influenciada por outras variáveis, a não ser dos seus valores passados. Este método é o mais simples e usual de previsão, e quando bem elaborado oferece bons resultados.

A fim de se montar um bom modelo de previsão, é necessário construir gráficos com os dados históricos para então possibilitar a identificação dos fatores que estão por trás das características da curva obtida, tais como, tendência, sazonalidade, variações irregulares e variações randômicas.

Em função de cada um desses fatores que influenciam os dados pode-se utilizar técnicas específicas para tratá-los, ou seja, existem técnicas para tratar a média, a tendência e a sazonalidade. A seguir, cada uma delas será descrita.

\subsection{Técnicas para previsão da média}

Essas técnicas fazem com que valores historicamente baixos e valores historicamente altos se combinem, gerando uma previsão média com menor variabilidade do que os dados originais. Outro ponto importante é que essas técnicas procuram privilegiar os dados mais recentes da série da série histórica, que normalmente representam melhor a situação atual. Essas técnicas funcionam bem quando os dados históricos variam em torno de uma média, porém ainda podem ser usadas quando existem pequenas variações graduais, ou em patamares, no nível dos dados (TUBINO, 2009).

As técnicas de previsão para a média mais utilizadas são a média móvel e a média exponencial móvel, detalhadas a seguir.

\section{a) Média Móvel}

A média móvel usa dados de um número predeterminado de períodos, normalmente os mais recentes, para gerar sua previsão. A cada novo período de previsão se substitui o dado mais antigo pelo mais recente. A média móvel pode ser obtida a partir da Equação 1.

$$
M m=\frac{\sum_{i=1}^{n} D_{i}}{n}
$$

onde:

Mm = média móvel de $\mathrm{n}$ períodos;

$\mathrm{D}_{\mathrm{i}}=$ demanda ocorrida no período i;

$\mathrm{n}$ = número de períodos; e 
$\mathrm{i}=$ índice d período $(\mathrm{i}=1,2,3, \ldots)$.

A grande vantagem do uso dessa técnica é a sua simplicidade operacional e facilidade de entendimento, porém, o que limita seu uso é a necessidade de armazenar um grande volume de dados, principalmente, quando o número de períodos (n) for grande. Além disso, ela fornece apenas a previsão para o período imediatamente posterior, e para os períodos futuros se usaria o mesmo valor, uma vez que há previsão de demandas médias. Recomenda-se o uso da média móvel em situações nas quais a demanda apresenta comportamento estável e a produtos não muito relevantes.

\section{b) Média exponencial móvel}

Na média exponencial móvel, o peso de cada dado decresce no tempo em progressão geométrica, ou de forma exponencial. Em sua forma de apresentação mais simples, cada nova previsão é obtida com base na previsão anterior, corrigido por um coeficiente de ponderação. A média exponencial móvel pode ser obtida a partir da Equação 2 (TUBINO, 2009).

$$
\mathrm{M}_{\mathrm{t}}=\mathrm{M}_{\mathrm{t}-1}+\alpha\left(\mathrm{D}_{\mathrm{t}-1}-\mathrm{M}_{\mathrm{t}-1}\right)
$$

sendo:

$\mathrm{M}_{\mathrm{t}}=$ previsão para o período $\mathrm{t}$;

$\mathrm{M}_{\mathrm{t}-1}=$ previsão para o período $\mathrm{t}-1$;

$\alpha=$ coeficiente de ponderação;

$\mathrm{D}_{\mathrm{t}-1}=$ demanda do período $\mathrm{t}-1$.

Segundo o autor supracitado o coeficiente de ponderação $(\alpha)$ é fixado pelo analista dentro de uma faixa de 0 a 1 . Quanto maior seu valor, mais rapidamente o modelo de previsão reagirá a uma variação real da demanda.

Para se tratar demandas médias, e ainda conseguir acompanhar movimentos pequenos de tendência ou de mudança de patamares, as previsões baseadas na média exponencial móvel são as mais utilizadas. Seu modelo exige a armazenagem de apenas três dados por item (a previsão passada, a demanda e o coeficiente de correlação), e a sua operação é de fácil entendimento. Contudo, como na média móvel, apresenta a desvantagem de fornecer previsão apenas para o período imediatamente posterior, sendo que para os períodos futuros se usaria o mesmo valor (TUBINO, 2009).

\subsection{Técnicas para previsão da tendência}


Tubino (2009) define tendência como o movimento gradual de longo prazo da demanda. Pode-se então calcular a estimativa desta tendência a partir da identificação da equação que descreva este movimento. A plotagem dos dados passados permitirá a identificação desta equação.

Existem duas técnicas importantes que podem ser utilizadas para resolver problemas de previsão de demanda com componentes de tendência linear: a equação linear para a tendência e o ajustamento exponencial para a tendência (TUBINO, 2009).

\section{a) Equação linear para a tendência}

A equação linear para tendência possui o formato da equação 3 (TUBINO, 2009, p. 23):

$$
Y=a+b x
$$

onde:

$\mathrm{Y}=$ Previsão de demanda para o período $\mathrm{x}$;

$\mathrm{a}=$ Ordenada à origem, ou intercessão no eixo dos $\mathrm{Y}$;

$\mathrm{b}=$ Coeficiente angular; $\mathrm{e}$

$\mathrm{x}=$ Período (partindo de $\mathrm{x}=0$ ) para previsão.

Empregando-se os dados históricos da demanda, os coeficientes b e a podem ser obtidos através das equações 4 e 5 (TUBINO, 2007, p.23).

$$
\begin{aligned}
& b=\frac{n\left(\sum X Y\right)-\left(\sum X\right)\left(\sum Y\right)}{n\left(\sum X^{2}\right)-\left(\sum X\right)^{2}} \\
& a=\frac{\sum Y-b\left(\sum X\right)}{n}
\end{aligned}
$$

onde: $\mathrm{n}=$ número de períodos observados.

\section{b) Ajustamento exponencial para a tendência}

A técnica do ajustamento exponencial para a tendência, ou também chamada de duplo ajustamento, é uma variação da técnica da média exponencial móvel e é usada para tratar demandas que apresentem tendências (TUBINO, 2009).

A aplicação da técnica consiste em fazer a previsão baseada em dois fatores: a previsão média exponencial móvel da demanda e uma estimativa exponencial da tendência. As equações 6, 7 e 8 representam estes elementos (TUBINO, 2009, p.25):

$$
P_{t+1}=M_{t}+T_{t}
$$

Sendo que:

$$
M_{t}=P_{t}+\alpha_{1}\left(D_{t}+P_{t}\right)
$$




$$
T_{t}=T_{t-1}+\alpha_{2}\left(\left(P_{t}-P_{t-1}\right)-T_{t-1}\right)
$$

onde:

$\mathrm{P}_{\mathrm{t}+1}=$ previsão da demanda para o período $\mathrm{t}+1$;

$\mathrm{P}_{\mathrm{t}}=$ previsão da demanda para o período $\mathrm{t}$;

$\mathrm{P}_{\mathrm{t}-1}=$ previsão da demanda para o período $\mathrm{t}-1$;

$\mathrm{M}_{\mathrm{t}}$ = previsão da média exponencial móvel da demanda para o período t;

$\mathrm{T}_{\mathrm{t}}=$ previsão de tendência exponencial móvel para o período $\mathrm{t}$;

$\mathrm{T}_{\mathrm{t}-1}=$ previsão de tendência exponencial móvel para o período $\mathrm{t}-1$;

$\alpha_{1}=$ coeficiente de ponderação da média;

$\alpha_{2}=$ coeficiente de ponderação da tendência; e

$\mathrm{D}_{\mathrm{t}}=$ demanda do período $\mathrm{t}$.

Para utilizar essa técnica de previsão da demanda, os valores dos coeficientes de ponderação são estipulados para corrigir os erros de previsão. Eles são usados com a mesma relação à media exponencial móvel, que devem ficar entre 0 e 1 .

\subsection{Técnicas para previsão da sazonalidade}

Tubino (2009) explica que a sazonalidade é caracterizada pela ocorrência de variações, para cima ou para baixo, em intervalos regulares na série temporal, e pode ser expressa na quantidade ou percentagem dos valores analisados. O valor aplicado sobre a média ou a tendência é conhecido como Índice de Sazonalidade (IS). A forma mais simples de considerar a sazonalidade nas previsões da demanda consiste em utilizar o último dado da demanda no período sazonal em questão, e assumi-lo como previsão para o próximo período semelhante. Se existir tendência, ela deverá ser adicionada ou retirada, do valor obtido. Porém, a forma mais usual de inclusão da sazonalidade nas previsões da demanda consiste em obter o índice de sazonalidade para os diversos períodos, empregando a média móvel centrada, e aplicá-los sobre o valor médio (ou tendência) previsto para o período em questão.

\section{a) Sazonalidade simples}

A sazonalidade simples é a técnica de previsão que consiste em obter o índice de sazonalidade para cada um dos períodos da série e aplicá-lo em cima da previsão da média em cada um desses períodos. Donatelli (2011) operacionaliza o cálculo da sazonalidade simples a partir da Equação 9.

$$
D_{P}=D_{M}+D_{M} *(I S-1)
$$


sendo:

$\mathrm{D}_{\mathrm{P}}=$ demanda prevista;

$\mathrm{D}_{\mathrm{M}}=$ demanda média; $\mathrm{e}$

IS = índice de sazonalidade.

\section{b) Sazonalidade com tendência}

Tubino (2009) afirma que quando a demanda apresentar sazonalidade com tendência há necessidade de se incorporar estas duas características no modelo de previsão. Para se fazer isso, devem-se empregar os seguintes passos:

1. Obter os índices de sazonalidade através da média móvel centrada;

2. Retirar o componente de sazonalidade da série de dados históricos, dividindo-os pelos correspondentes índices de sazonalidade;

3. Com esses dados, desenvolver uma equação que represente o componente de tendência; e

4. Com a equação da tendência, fazer a previsão da demanda e multiplicá-la pelo índice de sazonalidade.

Novamente, Donatelli (2011) operacionalizou o cálculo da sazonalidade com tendência a partir da Equação 10.

$$
\mathrm{D}_{\mathrm{P}}=\mathrm{T}+(\mathrm{T} *(\mathrm{IS}-1))
$$

sendo:

$\mathrm{D}_{\mathrm{P}}=$ demanda prevista;

$\mathrm{T}=$ tendência; $\mathrm{e}$

IS = índice de sazonalidade.

\subsection{Definição do modelo}

A fim de definir qual o melhor método de previsão é necessário que parâmetros sejam utilizados ou estimados. Donald et al. (2002) explica que os erros de previsão podem ser medidos em bases absolutas ou relativas. Embora o erro de previsão possa ser definido como a diferença entre a demanda real e a sua previsão, uma definição mais precisa se torna necessária para cálculo e comparação. Assim, ao utilizar a diferença entre o valor real e o valor previsto podem-se encontrar valores positivos e negativos e na soma dos erros estes valores se anularem, ocultando erros significativos na série. Uma saída seria utilizar o valor absoluto do erro (ABS) ou o desvio médio absoluto (MAD). 
O Mean Absolute Deviation (MAD), ou Desvio Médio Absoluto, multiplicado por 4 consiste no limite superior e -4 no inferior do gráfico de controle, do Controle Estatístico do Processo, que equivalem a três desvios-padrões. Caso o valor previsto exceda esses limites, ações corretivas devem ser tomadas. Quando o valor previsto ultrapassa o limite superior, os gestores devem estar atentos a essas variações e adequar a produção para atender esse mercado. Quando o valor previsto ultrapassa o limite inferior, os gestores devem efetuar alterações que promovam a venda dos seus produtos. A Equação 11 demonstra o cálculo do valor do MAD (TUBINO, 2009).

$$
M A D=\frac{\sum\left|D_{\text {atual }}-D_{\text {previta }}\right|}{n}
$$

sendo:

$\mathrm{D}_{\text {atual }}=$ demanda ocorrida no período;

$\mathrm{D}_{\text {prevista }}=$ demanda prevista no período; $\mathrm{e}$ $\mathrm{n}$ = número de períodos.

\section{Metodologia}

\subsection{Classificação da pesquisa}

Para a classificação da pesquisa, toma-se como base a taxionomia apresentada por Vergara (2004), que a qualifica em relação a dois aspectos: quanto aos fins e quanto aos meios.

Quanto aos fins, a pesquisa é aplicada, pois propõe a solução de um problema específico que é o ajustamento do modelo de previsão que melhor explique os dados de vendas de gasolina no Espírito Santo.

Quanto aos meios, a pesquisa será bibliográfica e documental. Bibliográfica, porque para a fundamentação teórico-metodológica do trabalho foi realizada investigação sobre os seguintes assuntos: Previsões, Tipos de Previsões, Previsão de Demanda, Etapas de um modelo de previsão e Técnicas de previsão. A investigação foi, também, documental, porque se valeu de planilhas extraídas do Portal Brasileiro de Dados Abertos, do Governo Federal. Nestas planilhas constam dados estatísticos sobre vendas de combustíveis. As planilhas demonstram as vendas realizadas pelas distribuidoras dos derivados combustíveis de petróleo e de álcool etílico hidratado, por produto, Unidade da Federação e Grande Região. As informações baseiam-se em dados declaratórios enviados à ANP pelas empresas responsáveis pela distribuição destes combustíveis e incluem as vendas propriamente ditas e o consumo próprio das empresas.

\subsection{Universo e amostra}


O universo da pesquisa é todo o histórico de vendas de gasolina no estado do Espírito Santo e a amostra os dados, da quantidade de gasolina vendida $\mathrm{em} \mathrm{m}^{3}$, mensais de 2000 a 2015.

\subsection{Coleta de dados}

Os dados foram coletados no Portal Brasileiro de Dados Abertos do Governo Federal.

\subsection{Tratamento dos dados}

A organização e a análise dos dados foram realizadas por meio software computacional Microsoft Office Excel. Obtida a planilha dinâmica que contém os dados de vendas, por produto e Unidade da Federação, pode-se extrair desta planilha os dados que se referem ao estado do Espírito Santo e a Gasolina C.

Dessa forma, pode-se gerar o gráfico que mostra a evolução das vendas de Gasolina C no estado do Espírito Santo, o gráfico de curva de tendência e o gráfico que faz a comparação entre as demandas real e prevista de gasolina pelos métodos Média Móvel, Média Exponencial Móvel, Equação Linear para a Tendência e Ajustamento Exponencial para a Tendência.

Por meio das facilidades do software Excel, ajustaram-se modelos, onde também é fornecido o valor de $\mathrm{R}^{2}$, para a obtenção do componente da tendência na previsão da sazonalidade com tendência.

Assim, construiu-se 6 planilhas para que as seguintes técnicas pudessem ser aplicadas: Média Móvel, Média Exponencial Móvel, Equação Linear para a Tendência, Ajustamento Exponencial para a Tendência, Sazonalidade Simples e Sazonalidade com Tendência.

Também se utilizou o software estatístico $\mathrm{R}$ para a análise da estatística descritiva proveniente dos dados de venda de gasolina no estado do Espírito Santo, de janeiro de 2000 a março de 2015.

As etapas seguidas neste trabalho para a previsão da demanda foram citadas por Tubino (2009), que consiste em coletar e analisar os dados históricos, para identificar e desenvolver a técnica de previsão da demanda que melhor se adapte ao setor petrolífero.

Para a escolha do melhor método, será feita análise do erro e da média absoluta dos erros (MAD).

\section{Resultados}

Neste capítulo foi realizada uma análise da série temporal e a seguir apresentadas e analisadas as previsões de demanda de gasolina no estado do Espírito Santo, entre o período de janeiro de 2000 a março de 2015. Para tanto, foram utilizadas as seguintes técnicas: média móvel, 
média exponencial móvel, tendência simples, ajustamento exponencial para tendência, sazonalidade simples e sazonalidade com tendência.

\subsection{Análise da série temporal}

Conhecer o comportamento da série temporal é imprescindível para determinar qual método de previsão obtém melhor ajuste. Na Tabela 1 pode-se observar a estatística descritiva proveniente dos dados de venda de gasolina no estado do Espírito Santo, de janeiro de 2000 a março de 2015.

Tabela 1 - Dados de vendas mensais de gasolina no ES (jan/00 a mar/15)

\begin{tabular}{cc}
\hline Média & 48260,14 \\
Desvio-padrão & 15224,36 \\
IQR & 22049,35 \\
$\mathbf{0 \%}$ & 29136,34 \\
$\mathbf{2 5 \%}$ & 36882,77 \\
$\mathbf{5 0 \%}$ & 41250,93 \\
$\mathbf{7 5 \%}$ & 58932,12 \\
$\mathbf{1 0 0 \%}$ & 92822,61 \\
\hline
\end{tabular}

Fonte: A autora

A partir da Tabela 1 percebe-se que a série apresenta 183 observações, variando de $29.136,34 \mathrm{~m}^{3}(\mathrm{jan} / 2002)$ a $92.822,61 \mathrm{~m}^{3}(\mathrm{dez} / 2014)$.

Ao observar o Gráfico 1, é possível notar que as vendas parecem decrescer até 2004, crescendo lentamente a partir desse momento até 2009, onde então parece crescer em tendência de forma mais substancial.

\subsection{Resultados das técnicas para a previsão da média}

\section{a) Média móvel}

Nessa técnica da previsão, o número de períodos (n) foi dividido da seguinte forma: três (Mm3), seis (Mm6), nove (Mm9) e doze (Mm12) meses. Na Tabela 2 estão os valores do erro acumulado e MAD, obtidos ao utilizar esses períodos.

Tabela 2 - Monitoramento da média móvel

\begin{tabular}{ccccc}
\cline { 2 - 5 } & Mm3 & Mm6 & Mm9 & Mm12 \\
\hline $\begin{array}{c}\text { Erro acumulado de jan de 2001 a } \\
\text { março de 2015 }\end{array}$ & $79.568,633$ & $154.283,074$ & $210.220,813$ & $-88.560 .483,469$ \\
MAD de jan de 2001 a mar/15 & 3113,147 & 3336,005 & 3339,260 & 517897,564 \\
\hline Fonte: A autora
\end{tabular}

Fonte: A autora

Ao observar o erro acumulado, o período que apresentou o menor valor foi o de 3 meses, isto pode ser explicado pois pequenos períodos permitem uma reação maior a mudanças da demanda, enquanto grandes períodos tratam a média de forma mais homogênea.

No que se refere ao MAD, o período de três meses também obteve o menor valor do erro. 
Dessa forma, o melhor modelo ajustado é o que utiliza o número de 3 períodos, como apresentado na Equação 12.

$$
M m_{3}=\frac{\sum_{i=1}^{\mathrm{g}} D_{\mathrm{i}}}{3}
$$

\section{b) Média exponencial móvel}

Nesta técnica de previsão, o coeficiente de ponderação $\alpha$ variou de 0,1 a 1 . Na Tabela 3 estão os valores do erro acumulado e MAD, obtidos ao utilizar esses coeficientes. 
Gráfico 1 - Vendas, pelas distribuidoras, de GASOLINA C (m3) no estado do Espírito Santo

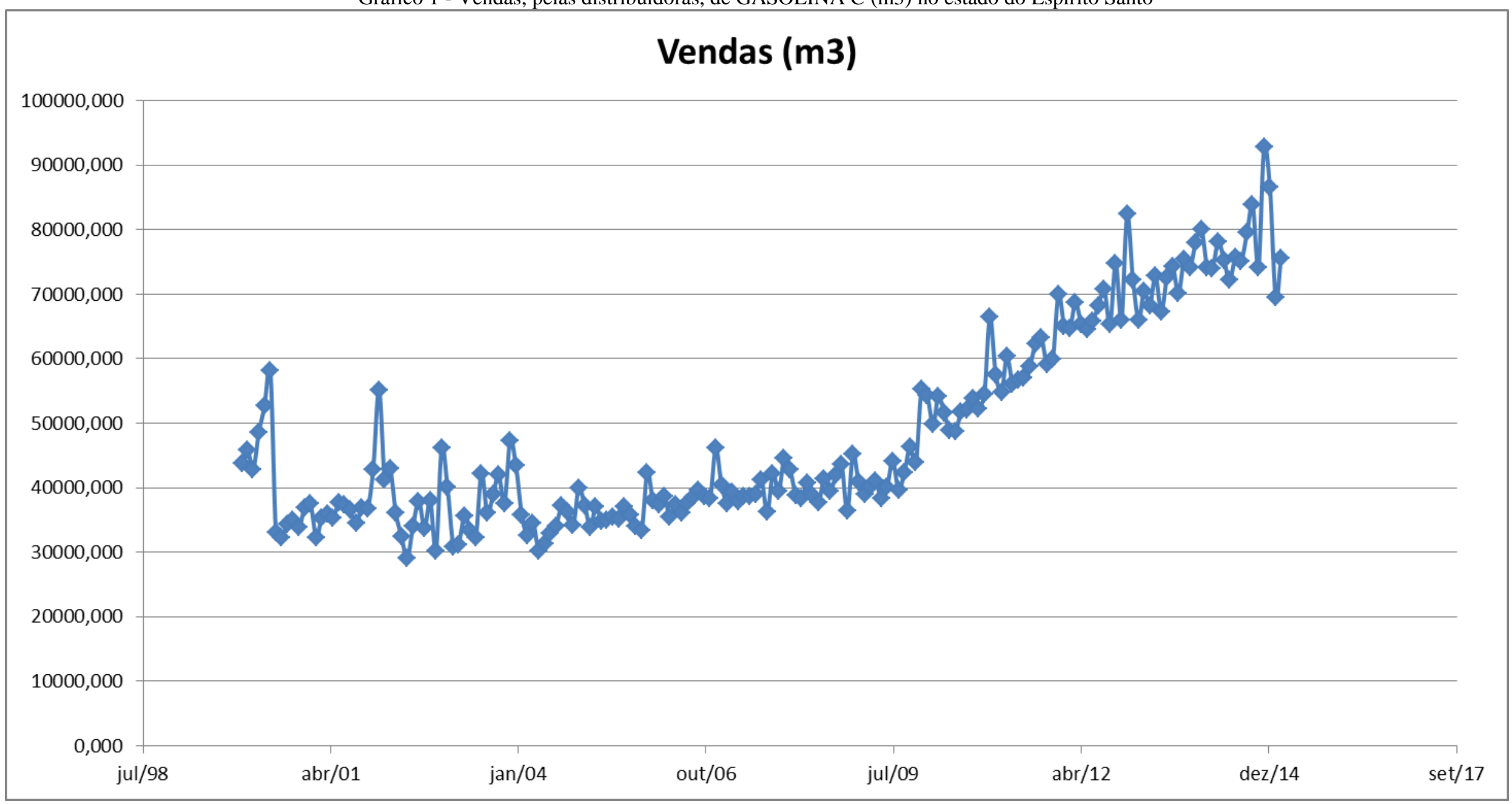

Fonte: A autora 
Tabela 3 - Monitoramento da Média Exponencial Móvel

\begin{tabular}{ccc}
\hline $\boldsymbol{\alpha}$ & Erro Acumulado & MAD \\
\hline $\mathbf{0 , 1}$ & $330.031,126$ & $4.256,979$ \\
$\mathbf{0 , 2}$ & $172.478,369$ & $3.426,578$ \\
$\mathbf{0 , 3}$ & $114.411,312$ & $3.271,075$ \\
$\mathbf{0 , 4}$ & $84.131,580$ & $3.267,591$ \\
$\mathbf{0 , 5}$ & $65.679,727$ & $3.305,005$ \\
$\mathbf{0 , 6}$ & $53.489,385$ & $3.367,077$ \\
$\mathbf{0 , 7}$ & $45.070,012$ & $3.469,494$ \\
$\mathbf{0 , 8}$ & $39.115,223$ & $3.599,139$ \\
$\mathbf{0 , 9}$ & $34.859,356$ & $3.734,511$ \\
$\mathbf{1 , 0}$ & $31.810,879$ & $3.882,218$ \\
\hline
\end{tabular}

Fonte: A autora

Dos coeficientes de ponderação testados, o escolhido foi o de 0,5 ; cujo erro acumulado foi de 65.679,727 e MAD igual a 3.305,005, como exposto na Equação 13.

$$
\mathrm{M}_{\mathrm{t}}=\mathrm{M}_{\mathrm{t}-1}+0,5\left(\mathrm{D}_{\mathrm{t}-1}-\mathrm{M}_{\mathrm{t}-1}\right)
$$

É possível observar que os valores obtidos no monitoramento deste coeficiente, não são os menores valores entre os coeficientes testados, porém ao comparar com os resultados dos demais coeficientes, o coeficiente de ponderação 0,50 possui os valores referentes ao monitoramento entre os menores, considerando assim que esse coeficiente apresentou melhor desempenho.

\subsection{Resultados das técnicas para a previsão da tendência}

\section{a) Equação linear para a tendência}

Ao aplicar esta técnica para a previsão da demanda, foi possível obter a Equação 14. Na Tabela 4 estão os valores do erro acumulado e MAD, obtidos ao utilizar a Equação 14.

$$
Y=46740,8945+16,51356 X
$$

Tabela 4 - Monitoramento da Equação Linear para a Tendência

\begin{tabular}{cc}
\hline & Equação Linear para a Tendência \\
\hline Erro acumulado & 0 \\
MAD & 12400,557 \\
\hline
\end{tabular}

Fonte: A autora

Esse modelo de previsão apresentou o valor de erro acumulado igual a 0 e MAD igual a $12.400,557$.

b) Ajustamento exponencial para a tendência 
Ao aplicar a técnica de ajustamento exponencial para a tendência, o coeficiente de ponderação da média $(\alpha 1)$ considerado foi 0,7 e o coeficiente de ponderação da tendência $(\alpha 2)$ foi 0,3 , que são os mesmos coeficientes utilizados por Tubino (2009). Porém, alteraram-se os valores dos coeficientes para que pudesse ser realizada uma análise de sensibilidade nos valores de erro acumulado e MAD, que podem ser observados na Tabela 5, a fim de identificar quais valores dos coeficientes promovem os valores mais precisos na previsão.

Tabela 5 - Monitoramento do Ajustamento Exponencial para a Tendência

\begin{tabular}{|c|c|c|}
\hline & Erro acumulado & MAD \\
\hline$\alpha 1=0,7$ e $\alpha 2=0,3$ & $-53761,671$ & 4186,444 \\
\hline$\alpha 1=0,8$ e $\alpha 2=0,3$ & $-53740,759$ & 4173,652 \\
\hline$\alpha 1=0,9$ e $\alpha 2=0,3$ & $-53140,955$ & 4208,609 \\
\hline$\alpha 1=0,8$ e $\alpha 2=0,4$ & $-56609,015$ & 4469,936 \\
\hline$\alpha 1=0,8$ e $\alpha 2=0,2$ & $-50611,669$ & 3911,447 \\
\hline$\alpha 1=0,8$ e $\alpha 2=0,1$ & $-46163,386$ & 3771,039 \\
\hline$\alpha 1=0,8$ e $\alpha 2=0$ & 51029,571 & 3674,089 \\
\hline
\end{tabular}

Fonte: A autora

Assim, pode-se observar na Tabela 4 que os valores dos coeficientes de $\alpha_{1}$ e $\alpha_{2}$ que miniminizão o erro acumulado e MAD são 0,8 e 0,1, respectivamente, que obteve o valor de $-46.163,386$ para o erro acumulado e 3.771,039 para o MAD. A equação referente a estes parâmetros, que melhor ajusta o modelo está explicitada na Equação 15.

$$
P_{t+1}=P_{t}+0,8\left(D_{t}-P_{t}\right)+T_{t-1}+0,1\left(\left(P_{t}-P_{t-1}\right)-T_{t-1}\right)
$$

\subsection{Resultados das técnicas para a previsão da sazonalidade}

\section{a) Sazonalidade simples}

Na técnica de previsão da sazonalidade simples, necessita-se obter o ciclo de sazonalidade. Dessa forma, criou-se uma tabela a fim de identificar qual a maior frequência no ciclo de sazonalidade. Pode-se então constatar que com um número de 28 repetições o ciclo de sazonalidade para a série de dados estudada é de 4 períodos, como apresenta a Tabela 6.

Tabela 6 - Frequência dos Ciclos de Sazonalidade

\begin{tabular}{cc}
\hline Ciclos de sazonalidade & Repetições \\
\hline 3 & 16 \\
4 & 28 \\
5 & 8 \\
6 & 4 \\
7 & 2 \\
\hline
\end{tabular}

Fonte: A autora 
$\mathrm{Na}$ Tabela 7 é apresentado o valor do erro acumulado e do MAD obtidos com a técnica de sazonalidade simples, utilizando o ciclo de sazonalidade igual a 4 períodos.

Tabela 7 - Monitoramento da Sazonalidade Simples

\begin{tabular}{cc}
\hline & Sazonalidade Simples \\
\hline Erro acumulado & 429839,416 \\
MAD & 12426,693 \\
\hline
\end{tabular}

Fonte: A autora

Nessa técnica o erro acumulado obtido foi de 429.839,416; além de apresentar o MAD igual a 12.426,693.

\section{b) Sazonalidade com tendência}

Para a obtenção da previsão da sazonalidade com tendência há a necessidade de se desenvolver uma equação que represente o componente da tendência. Dessa forma, ajustaram-se modelos por meio das facilidades do software Excel, onde também é fornecido o valor de $\mathrm{R}^{2}$, que podem ser observados na Tabela 8.

Tabela 8 - Equações que representem a Tendência

\begin{tabular}{ccc}
\hline Tipo de tendência & Equação & $\mathbf{R}^{\mathbf{2}}$ \\
\hline Linear & $\mathrm{y}=246,89 \mathrm{x}+27727$ & $\mathrm{R}^{2}=0,6793$ \\
Exponencial & $\mathrm{y}=31708 \mathrm{e}^{0,0046 \mathrm{x}}$ & $\mathrm{R}^{2}=0,6851$ \\
Logarítmica & $\mathrm{y}=9137,7 \ln (\mathrm{x})+11800$ & $\mathrm{R}^{2}=0,3003$ \\
Polinomial (ordem 2) & $\mathrm{y}=3,0349 \mathrm{x}^{2}-311,54 \mathrm{x}+44946$ & $\mathrm{R}^{2}=0,9085$ \\
Polinomial (ordem 3) & $\mathrm{y}=-0,0046 \mathrm{x}^{3}+4,3087 \mathrm{x}^{2}-405,54 \mathrm{x}+46407$ & $\mathrm{R}^{2}=0,9096$ \\
Polinomial (ordem 4) & $\mathrm{y}=-0,0002 \mathrm{x}^{4}+0,0764 \mathrm{x}^{3}-5,2935 \mathrm{x}^{2}-10,413 \mathrm{x}$ & $\mathrm{R}^{2}=0,9151$ \\
& +42682 & \\
Polinomial (ordem 5) & $\mathrm{y}=-6 \mathrm{E}-06 \mathrm{x}^{5}+0,0023 \mathrm{x}^{4}-0,3431 \mathrm{x}^{3}+23,775 \mathrm{x}^{2}$ & $\mathrm{R}^{2}=0,9226$ \\
Polinomial (ordem 6) & $\mathrm{y}=6 \mathrm{E}-08 \mathrm{x}^{6}-4 \mathrm{E}-05 \mathrm{x}^{5}+0,0096 \mathrm{x}^{4}-1,0621 \mathrm{x}^{3}+$ & \\
& $57,078 \mathrm{x}^{2}-1405,8 \mathrm{x}+50479$ & $\mathrm{R}^{2}=0,9246$ \\
Potência & $\mathrm{y}=23576 \mathrm{x}, 1693$ & $\mathrm{R}^{2}=0,3046$ \\
\hline
\end{tabular}

Fonte: A autora

O modelo escolhido foi o Polinomial de ordem 6 pois o seu valor de $\mathrm{R}^{2}(0,9246)$ mostra a melhor aderência entre os dados reais e a curva de previsão, como demonstra o Gráfico 2.

Na Tabela 9 é apresentado o valor do erro acumulado e do MAD obtidos com a técnica de sazonalidade com tendência.

Tabela 9 - Monitoramento da Sazonalidade com Tendência

\begin{tabular}{cc}
\hline & Sazonalidade com Tendência \\
\hline Erro acumulado & $-1762024650455,460$ \\
MAD & 9628550340,467 \\
\hline Fonte: A autora &
\end{tabular}

Fonte: A autora 
Nessa técnica o erro acumulado obtido foi de $-1762024650455,460$; além de apresentar o MAD igual a 9628550340,467.

Gráfico 2- Curva de tendência e equação

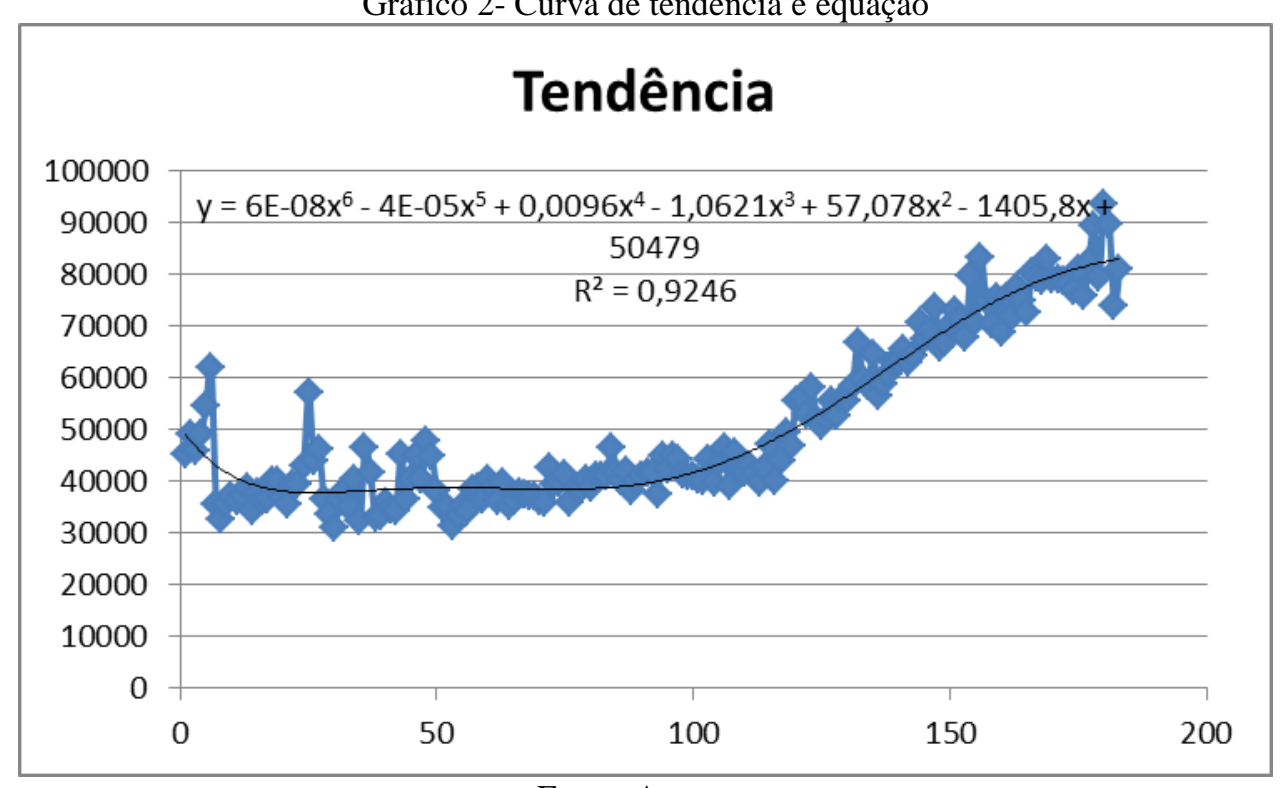

Fonte: A autora

\subsection{Definição do modelo}

Ao testar os modelos de previsão da demanda propostos pelo trabalho, na série temporal das vendas, pelas distribuidoras, da Gasolina C (m3) no Espírito Santo, foi possível obter os parâmetros referentes ao erro acumulado e MAD, com o objetivo de escolher o melhor modelo. Na Tabela 10 estão os valores que auxiliam no monitoramento das técnicas de previsão.

Tabela 10 - Monitoramento das Técnicas de Previsão

\begin{tabular}{ccc} 
& Erro acumulado & MAD \\
\cline { 2 - 3 } Média Móvel $(\mathbf{M m 3})$ & $79.568,633$ & $3.113,147$ \\
Média Exponencial Móvel $(\boldsymbol{\alpha}=\mathbf{0 , 5})$ & $65.679,727$ & $3.305,005$ \\
\hline Equação Linear para a Tendência & 0 & $12.400,557$ \\
Ajustamento Exponencial para a & $-46.163,386$ & $3.771,039$ \\
Tendência & & $12.426,693$ \\
Sazonalidade Simples & $429.839,416$ & $9.628 .550 .340,467$ \\
\hline Sazonalidade com Tendência & $-1.762 .024 .650 .455,460$ &
\end{tabular}

Fonte: A autora

Levando-se em consideração o erro acumulado os métodos que apresentaram maior precisão foram, em ordem decrescente, a Equação Linear para a Tendência, o Ajustamento Exponencial para a Tendência e a Média Exponencial Móvel. 
Enquanto os métodos que apresentaram menor MAD foram, em ordem decrescente, a Média Móvel, a Média Exponencial Móvel e o Ajustamento Exponencial para a Tendência.

A técnica de Equação Linear para a Tendência permite realizar previsão de demanda para qualquer período futuro, porém quanto mais distante o período a ser previsto menos exata será a previsão.

Já o método Ajustamento Exponencial para a Tendência consiste em uma adaptação do método da média móvel exponencial, possuindo como vantagem a maior precisão das previsões quando há tendência. Porém este método possui a desvantagem de realizar previsão apenas para o período imediatamente posterior.

Com relação às técnicas para a previsão de demanda que utilizam a média, elas são recomendadas para séries que possuem pouca variação, ou seja, para mercados mais estáveis e para produtos menos relevantes, o que não é o caso da série analisada. Esses modelos ainda apresentam a desvantagens de realizar a previsão para o período imediatamente posterior.

No Gráfico 3 pode ser visualizada a comparação entre a Média Móvel, Média Exponencial Móvel, Tendência Linear e Ajustamento Exponencial para a Tendência.

Percebe-se no Gráfico 3 que, apesar de apresentar o menor erro, a linha de tendência linear não acompanha o movimento da linha da demanda real de gasolina, ou seja, este método não acompanha o movimento da demanda ao longo dos períodos.

As técnicas para a previsão de demanda que utiliza à média apresentaram valor do MAD relativamente menor, porém os valores referentes ao erro acumulado foram maiores quando comparados à técnica de Ajustamento Exponencial para a Tendência.

Dessa forma, conclui-se que dentre os métodos estudados os dois principais modelos são a Média Exponencial Móvel e o Ajustamento Exponencial para a Tendência. 
Gráfico 3 - Demandas real e prevista de gasolina pelos métodos Média Móvel, Média Exponencial Móvel, Equação Linear para a Tendência e Ajustamento Exp. para a Tendência.

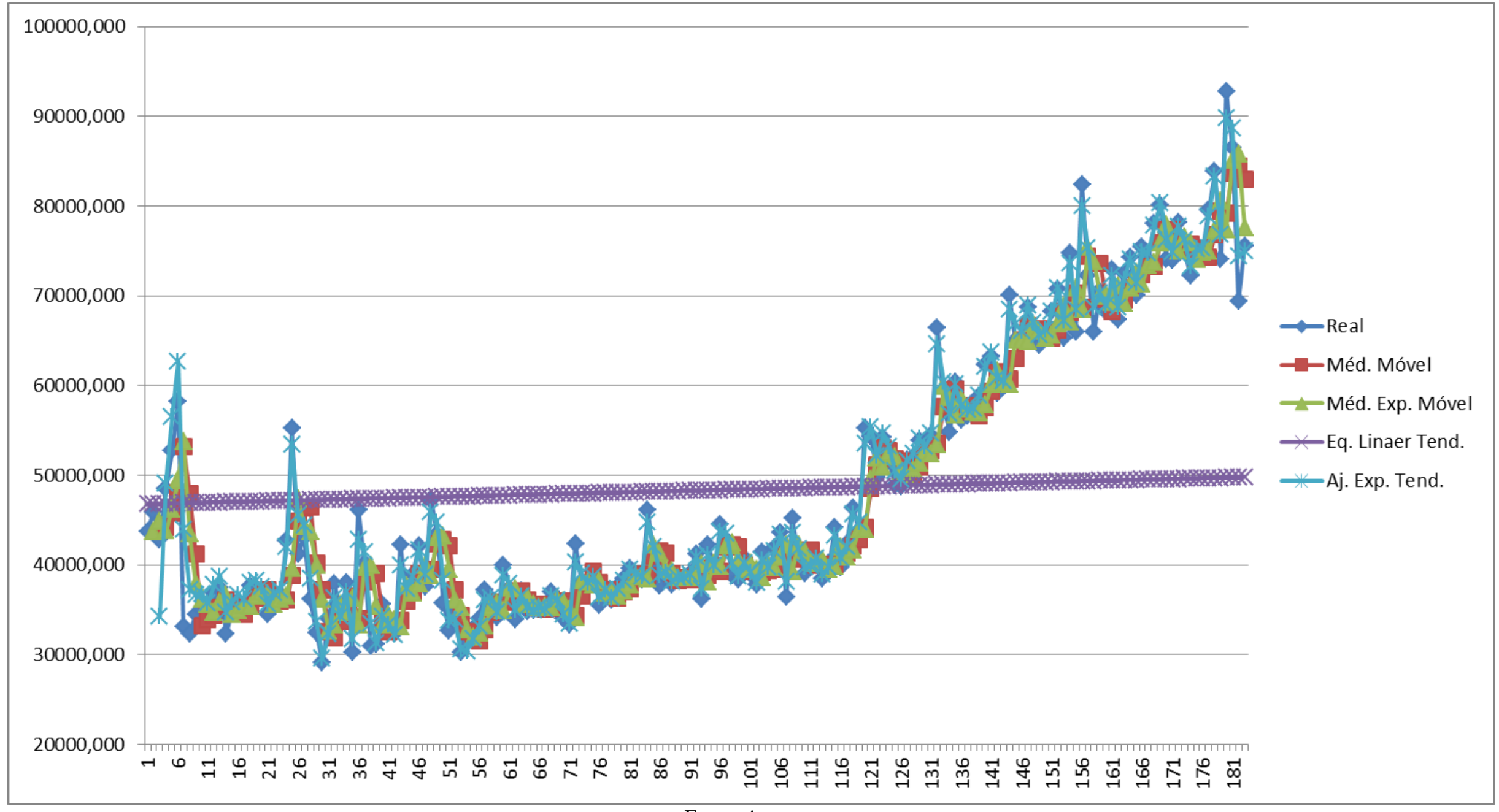

Fonte: A autora 
Na Tabela 11 estão apresentadas as previsões para os meses de abril, maio, junho e julho de 2015, utilizando esses dois modelos, bem como as vendas reais para esses quatro meses.

Tabela 11 - Comparação entre as vendas reais e as previsões para os meses de abril, maio, junho e julho de 2015 , utilizando Média Exponencial Móvel e Ajustamento Exponencial para a Tendência

\begin{tabular}{ccccc} 
& Abril/2015 & Maio/2015 & Junho/2015 & Julho/2015 \\
\hline $\begin{array}{c}\text { Média Exponencial Móvel } \\
\text { (Previsões) }\end{array}$ & $76.584,485$ & $76.938,833$ & $74.186,667$ & $74.062,989$ \\
$\begin{array}{c}\text { Ajustamento Exponencial para a } \\
\text { Tendência (Previsões) } \\
\text { Vendas (Reais) }\end{array}$ & $74.970,530$ & $76.556,895$ & $72.373,034$ & $73.130,319$ \\
\hline
\end{tabular}

Fonte: A autora

Com os dados da Tabela 11 pôde-se plotar o Gráfico 4.

Gráfico 4 - Demandas real e prevista de gasolina pelos métodos Média Exponencial Móvel e Ajustamento Exp. para a Tendência, para os meses de abril, maio, junho e julho de 2015.

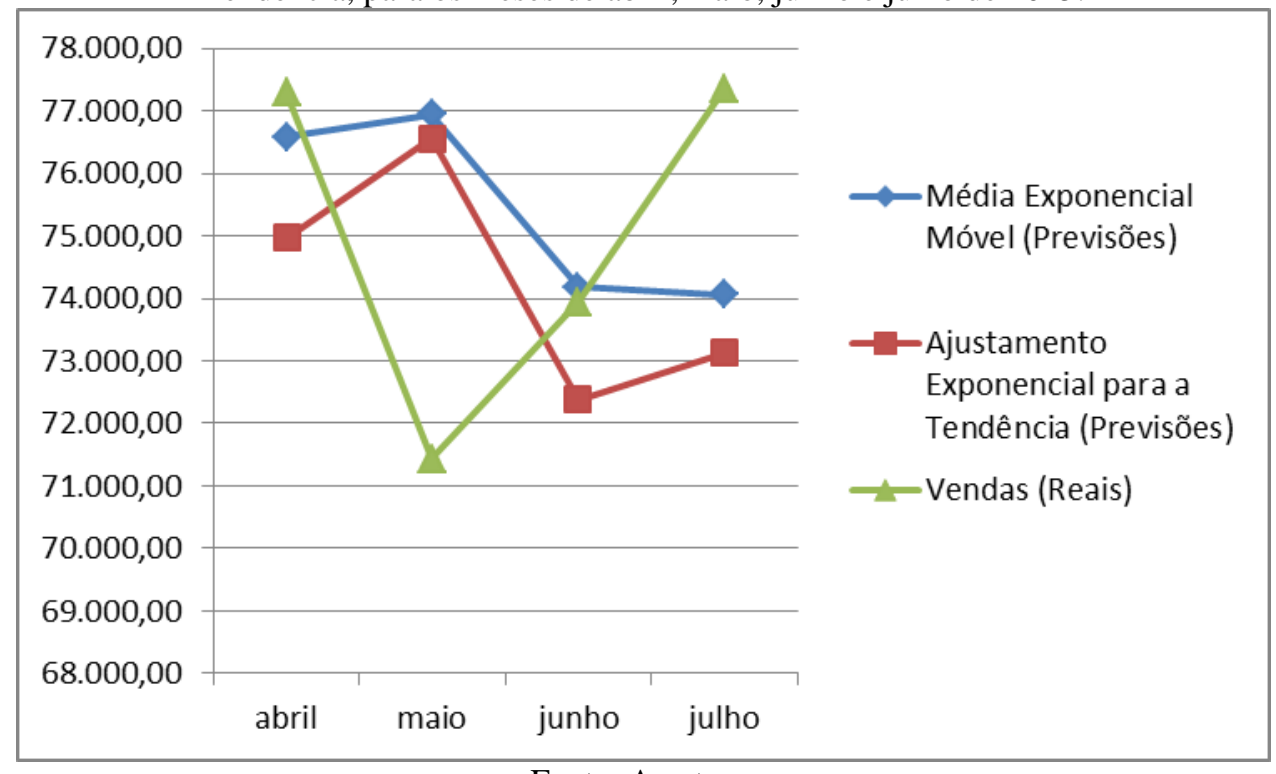

Fonte: A autora.

A partir da Tabela 11 e do Gráfico 4, pode-se constatar que a técnica Média Exponencial Móvel reage um pouco mais rápido a um aumento repentino da demanda. Porém a diferença nas previsões dos dois modelos expostos na Tabela 11 não foi muito significativa, o que também ressalta a importância do modelo ajustado por meio do Ajustamento Exponencial para a Tendência.

Visto que previsões com os dois melhores modelos ajustados puderam ser realizadas, podese calcular o Erro Acumulado à medida que os modelos foram alimentados, ou seja, calculou-se o Erro Acumulado para cada mês previsto (Tabela 12).

Da mesma forma, visto que previsões dos dois melhores modelos ajustados puderam ser realizadas, pode-se calcular o MAD para cada mês previsto (Tabela 13). 
Tabela 12 - Erro acumulado para os meses de abril, maio, junho e julho de 2015

\begin{tabular}{ccc}
\hline Erro acumulado até & Média Exponencial Móvel $(\boldsymbol{\alpha}=\mathbf{0 , 5})$ & $\begin{array}{c}\text { Ajustamento Exponencial } \\
\text { para a Tendência }\end{array}$ \\
\hline Abril de 2015 & $66.388,425$ & $-43.840,734$ \\
Maio de 2015 & $60.884,091$ & $-48.963,130$ \\
Junho de 2015 & $60.636,736$ & $-47.396,853$ \\
Julho de 2015 & $63.923,167$ & $-43.177,753$ \\
\hline
\end{tabular}

Fonte: A autora

Tabela 13 - MAD para abril, maio, junho e julho de 2015

\begin{tabular}{ccc}
\hline MAD & Média Exponencial Móvel $(\boldsymbol{\alpha}=\mathbf{0 , 5})$ & $\begin{array}{c}\text { Ajustamento Exponencial } \\
\text { para a Tendência }\end{array}$ \\
\hline Abril de 2015 & $3.290,818$ & $3.763,036$ \\
Maio de 2015 & $3.302,848$ & $3.770,505$ \\
Junho de 2015 & $3.286,332$ & $3.758,460$ \\
Julho de 2015 & $3.286,332$ & $3.760,964$ \\
\hline
\end{tabular}

Fonte: A autora

A partir da Tabela 12 percebe-se que o Erro Acumulado é menor em módulo para o modelo que utiliza o Ajustamento Exponencial para a Tendência. Já analisando a Tabela 13, é possível concluir que o MAD é menor para o modelo que utiliza a Média Exponencial Móvel. Porém, a diferença existente no cálculo dos erros não invalida nenhum dos modelos ajustados, ao contrário ressalta a importância dos dois modelos ajustados, o que utiliza a Média Exponencial Móvel e o que utiliza o Ajustamento Exponencial para a Tendência.

\section{Conclusão}

O presente trabalho caracterizou o comportamento da demanda de gasolina $\mathrm{C}\left(\mathrm{m}^{3}\right)$ no Espírito Santo ao longo de toda a série temporal analisada, e assim, determinou qual a melhor técnica de previsão de demanda para o caso em estudo.

Dentre as características que caracterizam uma série temporal, pode-se concluir que a demanda de gasolina estudada não apresenta a componente sazonalidade, visto que as técnicas de previsão Sazonalidade Simples e Sazonalidade com Tendência apresentaram os piores valores para o erro acumulado e o MAD, que são os parâmetros utilizados nesta pesquisa a fim de identificar qual o melhor método de previsão.

Quanto ao somatório dos erros, o modelo que mais se adequou à série temporal da empresa foi o de tendência linear, com erro igual a zero. Porém ao analisar o Gráfico 3 percebeu-se que, apesar de apresentar o menor erro, a linha de tendência linear não acompanha o movimento da linha da demanda real de gasolina, ou seja, este método não acompanha o movimento da demanda ao longo dos períodos. Ou seja, o que de fato está ocorrendo é que os erros estão se anulando. Prevendo a demanda por esta técnica, ocorrerá a formação de estoques em um período, e colocação deste estoque em períodos seguintes. Cabe, portanto avaliar o quanto este carregamento de estoques implica em aumento dos custos do produto, para as empresas. 
Levando em consideração o MAD os modelos que obtiveram melhor precisão foram as técnicas para a previsão de demanda que utiliza à média, porém os valores referentes ao erro acumulado foram maiores quando comparados à técnica de Ajustamento Exponencial para a Tendência.

Dessa forma, conclui-se que dentre os métodos estudados as principais técnicas de previsão, das vendas de Gasolina C $\left(\mathrm{m}^{3}\right)$ no Espírito Santo, foram a Média Exponencial Móvel e o Ajustamento Exponencial para a Tendência.

Cada método possui sua particularidade, sendo necessário conhecimento a respeito da série que se deseja prever e das características de cada método.

Ao analisar a série temporal da demanda de gasolina $\mathrm{C}\left(\mathrm{m}^{3}\right)$ no Espírito Santo, foi possível identificar três padrões: as vendas parecem decrescer até 2004; de 2004 até 2009 ela cresce lentamente; e a partir deste ponto começa a crescer em tendência de forma mais substancial.

Assim, em pesquisas futuras, podem ser estudados outros métodos de previsão, que possam vir a apresentar melhores resultados que os modelos estudados neste trabalho, além de poder ser realizadas análises utilizando-se métodos de previsão a partir da divisão do estudo em três séries, por exemplo, de 2000 à 2004, de 2004 à 2009, e por fim, de 2009 à 2015, como foi justificado no parágrafo anterior.

Por fim, outra sugestão para trabalhos futuros, é a aplicação de métodos de previsão de demanda para os produtos e estados que não foram analisados neste trabalho.

\begin{abstract}
Demand forecasting has become a major factor in company management, revealing itself as a strategic alternative to cope with demand fluctuations and thus avoid losses. The oil industry has great importance for the national economy, contributing to the generation of direct and indirect jobs. Therefore, studies on the consumption of petroleum derived fuels assist in the projections and plans in this sector. In addition to the sales forecast it is an important management tool. Thus, this article aims to set a demand forecasting model, better suited to demand for gasoline in the state of Espírito Santo, based on monthly consumption data for the period from January 2000 to March 2015. To carry out this study was first presented a literature review of the main types and demand forecasting techniques. Subsequently employed in quantitative demand forecasting techniques (moving average, exponential moving average, linear equation for the trend, exponential adjustment to the trend, simple seasonal and seasonal prone), and through the parameters for the accumulated error and Mean Absolute Deviation (MAD), compared the methods, and could be identified that the main forecasting techniques were Exponential Mobile Average and Exponential Adjustment to the trend.
\end{abstract}

Key-words: demand forecast; gasoline; trend.

\title{
Referências
}

BRUNI, A. L. Estatística Aplicada à Gestão Empresarial. São Paulo: Atlas, 2007. 
CAVAlHeIRO, D. Método de Previsão de Demanda Aplicada ao Planejamento da Produção de Indústrias de Alimentos. Dissertação (Mestrado em Engenharia) - Programa de Pós-Graduação em Engenharia Mecânica, Universidade Federal de Santa Catarina, Florianópolis, 2003.

CORRÊA, H. L.; CORRÊA, C. A. Administração de produção e operações: manufatura e serviços: uma abordagem estratégica. São Paulo: Atlas, 2007.

DONALD J., BOWERSOX, M.; BIXBY C., DAVID J. C. Gestão logística de cadeias de suprimentos. São Paulo: Artmed, 2002.

DONATElli, J. S. Análise e Previsão da Demanda Externa de Celulose e Papel no Brasil. Monografia (Graduação) - Departamento de Engenharia Florestal, Universidade Federal do Espírito Santo, Jerônimo Monteiro, 2011.

HAIZER, J.; RENDER, B. Administração de Operações. Rio de Janeiro: LTC, 2001.

MANCUSO, A. C. B.; WERNER, L. Estudo dos Métodos de Previsão de Demanda Aplicado em uma Empresa de Auditorias Médicas. Revista Ingeniería Industrial, año. 13, n. 1, 99-111, 2014.

PEINADO, J.; GRAEMEL, A. R. Administração da Produção: Operações Industriais e de Serviços. Curitiba: UnicenP, 2007.

SLACK, N.; CHAMBER, S.; HARDLAND, C.; HARRISON, A. e JOHNSTON, R. Administração da Produção. São Paulo: Atlas, 2002.

TUBINO, D. F. Planejamento e controle da produção. São Paulo: Atlas, 2009.

VERGARA, S.C. Projetos e relatórios de pesquisa em administração. Atlas, São Paulo, 2004.

\section{Dados dos autores:}

\section{Nome Completo: Noéle Bissoli Perini}

Filiação institucional: Universidade Federal do Espírito Santo

Função ou cargo ocupado: Mestranda

Endereço completo para correspondência: Rodovia BR 101, km 65, 2121, Santo Antônio - São

Mateus, 29941-510, ES - Brasil

Telefones para contato: (27) 99232-5235, ou (27) 99816-2575

e-mail:noeleperini@hotmail.com

\section{Nome Completo: Adelmo Inácio Bertolde}

Filiação institucional: Departamento de Estatística da Universidade Federal do Espírito Santo

Função ou cargo ocupado: Professor doutor em estatística

Endereço completo para correspondência: Departamento de estatística da UFES/CCE/UFES. Av.

Fernando Ferrari , n: 514, Goiabeiras, Vitória - ES.

Telefones para contato: (27) 4009-2481, ou (27) 9941-8575

e-mail: adelmoib@gmail.com 
Submissão: 22/09/2015

Aceito: 06/03/2016 